\title{
Breast Cancer and Multiple Primary Malignant Tumors: Case Report and Review of the Literature
}

\author{
ALESSANDRO DE LUCA ${ }^{1}$, FEDERICO FRUSONE ${ }^{1}$, MASSIMO VERGINE ${ }^{1}$, ROSARIO COCCHIARA ${ }^{2}$, \\ GIUSEPPE LA TORRE ${ }^{2}$, LAURA BALLESIO $^{3}$, MASSIMO MONTI $^{1}$ and MARIA IDA AMABILE ${ }^{1}$ \\ ${ }^{1}$ Department of Surgical Sciences, Sapienza University of Rome, Rome, Italy; \\ ${ }^{2}$ Department of Public Health and Infectious Diseases, Sapienza University of Rome, Rome, Italy; \\ ${ }^{3}$ Department of Radiology, Anatomo-Pathology and Oncology, Sapienza University of Rome, Rome, Italy
}

\begin{abstract}
Multiple primary malignant neoplasms are multiple tumors with different pathogenetic origin. They may be synchronous or metachronous. The management of these conditions represents an interesting clinical scenario. A crucial aspect is the decision regarding which tumor to treat initially, and how to schedule further treatments according to individual tumor risk. This process involves a multidisciplinary physician team to ensure favorable outcomes. We describe a case report of a female patient affected by primary synchronous tumors of the breast and pectoral skin, which raised a series of diagnostic, etiological and therapeutic issues persuading us to carry out a critical review of the literature.
\end{abstract}

Multiple primary malignant neoplasms (MPMNs), or multiple primary cancers, are defined as multiple tumors with different pathogenetic origin. The phenomenon of MPMN was first described by Billroth at the end of the 19th century (1) and since then several cases of double or even triple primary malignant neoplasms have been reported (1-3). Neoplasms can be synchronous or metachronous and may appear in a single organ or in multiple organs concurrently. According to Moertel (2), synchronous neoplasms are defined as those that occur within 6 months from the diagnosis of a previous malignant tumor, and metachronous neoplasms are defined as neoplasms that appear 6 months after the first diagnosed tumor (2). Despite many changes in the definition have been proposed, the diagnosis of MPMNs that is widely accepted is based on the criteria

This article is freely accessible online.

Correspondence to: Maria Ida Amabile, MD, PhD, Department of Surgical Sciences, Sapienza University of Rome, viale Regina Elena, 324, 00161 Rome, Italy. Tel: +390649975569, e-mail: mariaida.amabile@uniroma1.it

Key Words: Breast cancer, multiple primary malignant neoplasms, solid tumors, review. described by Warren and Gates (3). These criteria require that: (i) each tumor must have a clear picture and histological confirmation of malignancy; (ii) each tumor must be topographically distinct and separated by healthy mucosa (at least $2 \mathrm{~cm}$ of normal mucosa between two tumors of the same region); (iii) the lesions must be not metastases of each other (3).

In this report, we describe our recent observation and treatment of a female patient affected by primary synchronous tumors of the breast and pectoral skin. This combination, to the best of our knowledge, has never been previously reported in the literature, and it raised a series of diagnostic, etiological and therapeutic issues persuading us to carry out a critical review of the literature.

\section{Case Report}

A 58-year-old postmenopausal woman, of Balkan origin which had settled in Rome for the past 5 years, was referred to the Department of Surgical Sciences, Sapienza University of Rome, Italy, for an erythematous, ulcerated lesion with necrotic areas of the chest wall involving the skin of the right breast (Figure 1). The patient gave written informed consent for use of her radiological imaging and photographic documentation. A detailed past medical history was collected, which resulted negative for primary comorbidities, genetic or environmental exposition to risk factors, as well as for family history of cancer.

Interestingly, the patient also presented crusts and ulceration on the right nipple, suffering by intense pain and bleeding from the nipple as well as from the necrotic areas of the chest wall. No weight loss, nor fatigue, fever or any other specific complaints were reported.

\section{Clinical and Diagnostic Investigations}

In the first instance, a mere cutaneous lesion was suspected and, therefore, a punch biopsy was performed, whose histological diagnosis documented a squamous cell carcinoma 
of the pectoral skin. Moreover, because of the ulcerations within the right nipple-areola complex, the patient underwent mammography and breast ultrasonographic imaging that documented the presence of multiple nodular opacities with irregular margins, variable diameter from 5 to $10 \mathrm{~mm}$ and calcifications in the external upper quadrant (Figure 2a). A highly suspicious axillary lymphadenopathy was also detected (Figure 2b). The imaging showed a multicentric breast disease with an associated suspicious in situ component, indicating the necessity of an ultrasound-guided breast coreneedle biopsy (at two different sites). The histological examination documented a grade 3 invasive ductal carcinoma. Considering the coexistence of a cutaneous squamous cell carcinoma, immunohistochemical analyses were performed to verify the primitiveness of the breast lesions. The results were the following: human milk factor was non-probative; CKMNF116 resulted positive; p63, estrogen receptor (ER), progesterone receptor $(\mathrm{PgR})$ and HER-2 neu receptor resulted negative. Therefore, according to the immunophenotype documented and the negativity of p63 expression, the primitiveness of breast tumor was confirmed. Staging investigations were performed and resulted negative for metastatic lesions.

Finally, to exclude a possible genetic predisposition to breast cancer, the patient was evaluated for the presence of $B R C A 1 / 2$ gene mutations, but none was detected.

\section{Treatment}

After discussing the clinical picture of the patient in a multidisciplinary team, the patient underwent neoadjuvant chemotherapy. Due to the presence of two different tumors, a more aggressive approach was chosen compared to a normal chemotherapy protocol used in the case of clinical T4 breast cancer.

Already during the second cycle of chemotherapy, the staging computed tomography showed that the neoplastic lesions of the right breast were no longer appreciable.

Breast re-evaluation by magnetic resonance imaging, after three chemotherapy cycles, showed an important dimensional reduction of the cutaneous and breast lesions with re-epithelialization of the ulcerated areas.

The patient completed the neoadjuvant chemotherapy with three more treatment cycles and then underwent a combined surgery of right mastectomy, ipsilateral axillary lymph nodes dissection, excision of the remaining cutaneous squamous cell carcinoma, and reconstruction with a split-thickness skin graft taken from the contralateral leg with an electric dermatome, to correct for the loss of substance (Figure $3 \mathrm{a}$ and $3 \mathrm{~b}$ ). The surgery lasted $105 \mathrm{~min}$ and the estimated blood loss was $100 \mathrm{ml}$. The patient was discharged in the third post-operative day in a good clinical condition.

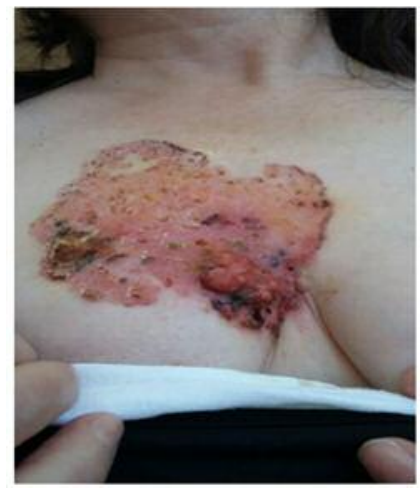

Figure 1. Ulcerated lesion with necrotic areas of the chest wall involving the skin of the right breast at the moment of the diagnosis.

The histopathological examination of the surgical specimen revealed a complete pathological response of the right breast carcinoma, documenting only a small residue of ductal carcinoma in situ, and a complete regression of the cutaneous squamocellular lesion.

No early neither late post-operative complications were documented, and the graft was well tolerated. Due to the absence of both cutaneous and breast infiltrating tumors, it was decided to keep the patient in follow-up without further oncological treatments.

The patient maintained a good performance status and she is continuing on a program of 6-month clinical and radiological follow-ups as recommended by the multidisciplinary team.

\section{Review of the Literature}

MPMNs incidence is estimated to be between $0.73 \%$ and $11.7 \%$ (4). Patients with a primary tumor have a $10 \%$ higher risk of developing a second tumor with respect to the general population $(5,6)$. Causes of MPMNs are not yet clear, but they may be related to four principal factors: intrinsic factors, extrinsic factors, genetic factors, and therapeutic factors.

Intrinsic factors include susceptibility, immune status, and endocrine and embryonic development. The loss of immune surveillance and immune defenses both congenital and acquired increases the possibility to develop a MPMNs (7).

Extrinsic factors are represented by environmental factors and personal lifestyle, including occupational diseases, long exposure to ultraviolet rays and industrial pollution (7). In addition, smoking and alcohol, and low physical activity are important carcinogenic factors.

Genetic factors may favor the development of MPMNs. Members of families with BRCA gene mutations have an increased risk of early onset breast and ovarian cancer (8).

Therapeutic factors, including carcinogenic radiation treatment and chemotherapy, may significantly increase the 

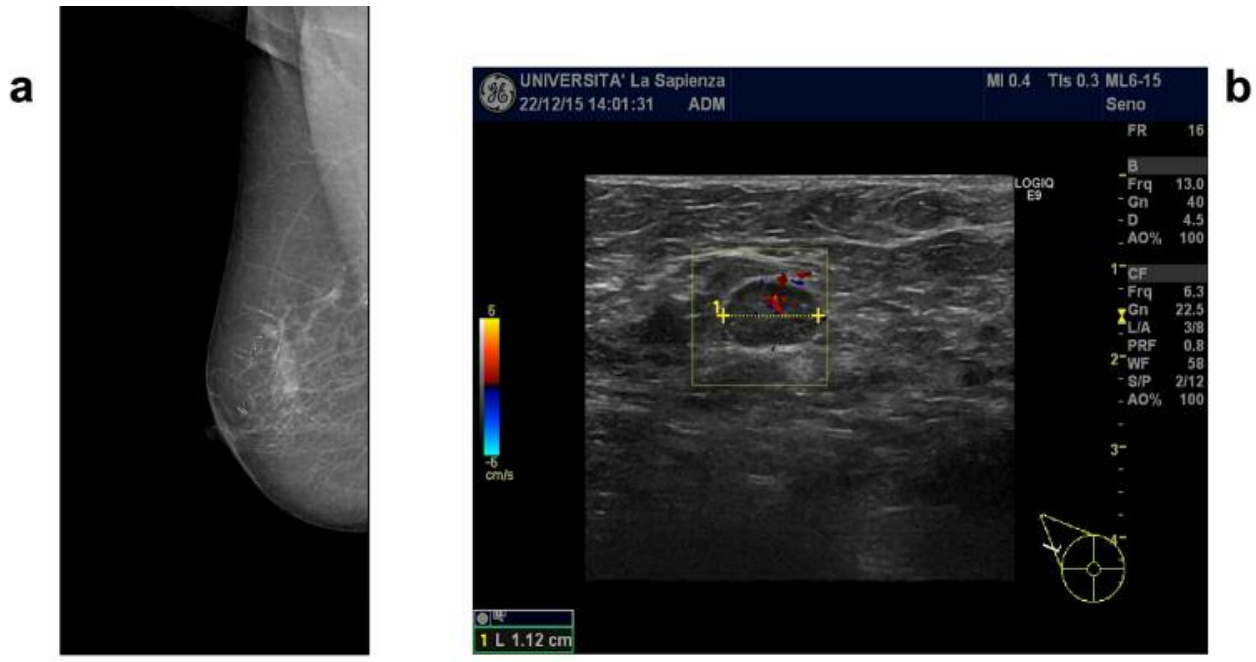

Figure 2. Breast radiological imaging. a) Mammographic imaging of the right breast at the moment of the diagnosis. b) Ultrasound imaging of the right axillary lymphoadenopathy documented at the moment of the diagnosis.
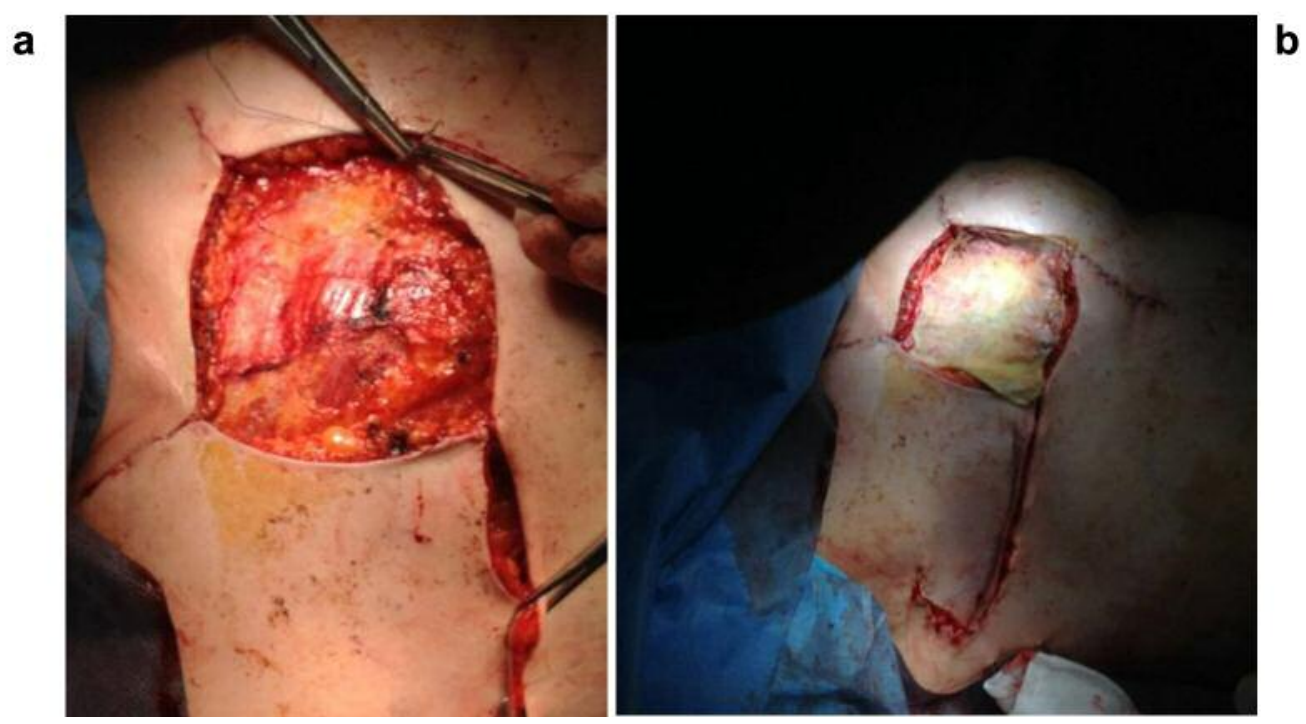

Figure 3. Breast reconstruction.

risk of developing MPMNs (9). It is surprising how chemotherapeutic agents might paradoxically have an "oncogenic" role, since on the one hand they increase survival, and on the other they may cause an acquired immunosuppression.

Spratt et al. (9) reported that new therapy protocols for cancer (i.e. alkylating agents, topoisomerase II inhibitor and/or radiotherapy) have caused increased rates of survival, determining a higher chance for patients to develop a second neoplasm and apparently increasing the incidence of cancer.
Above all, in the case of metachronous tumors, the main cause of the development of a second tumor is the acquired immunosuppression. Several immunosuppressive and/or cytotoxic agents are potential carcinogens and may either have a direct oncogenic effect or play a role as cofactor in oncogenesis (9).

In our review we considered all the published case reports of MPMNs where breast cancer is present and have evaluated the correlation of the different single tumors with breast cancer. 
Table I shows a summary of the previous studies describing MPMNs.

Breast cancer and skin cancer. Twelve cases of tumors involving the skin and the breast in the same person have been described (10-21); in particular, only two cases $(11,12)$ presented the coexistence of squamous cell carcinoma and breast cancer, similarly to our case, which turns out to be the third case report documented since 1980. As discussed by Pastore et al. (12), our patient presented a synchronism of a wide squamous cell carcinoma of the right breast synchronous to a right breast cancer.

The most frequent described MPMN is breast cancer and melanoma (13-20).

This association is not surprising, because several genetic and hormonal factors are responsible for the association between breast cancer and melanoma, in particular the germ-cell mutations or somatic mutations in genes such as BRCA2 (22).

Breast cancer and gastro-intestinal cancer. The association between breast cancer and gastro-intestinal cancer may be considered as a sporadic or germinal association (23-46). While for tumors of the high gastro-intestinal tract, such as esophagus, stomach and liver cancer, the association with breast cancer is probably sporadic ( 8 cases have been described) (14, 23-29), in case of colorectal cancer several studies emphasized the coexistence of common predisposing extrinsic and genetic factors.

Breast cancer and colon cancer represent primary tumors with high incidence, while metastatic breast cancer to the colon is a rare entity with the exception of the lobular histotype breast carcinoma which has resulted to be the most common histological type of breast cancer that metastasizes to the colon (47).

Breast carcinoma and nervous system tumors. The association between breast cancer and primitive brain tumor lesions is weak and less frequent $(16,48,49)$.

The presence of a breast cancer and a brain lesion always needs further investigation aimed at diagnosing the primitiveness of the single lesions, considering that breast cancer appears to be the second leading cause of brain metastasis (50).

Less frequent but often metachronous lesions are, instead, the associations between breast cancer and peripheric nervous system tumors (51-53).

Breast cancer and hematologic tumors. Breast cancer patients may develop, with a higher risk with respect to the normal population, secondary malignancies, including leukemia and non-Hodgkin's lymphoma (54). The second tumor is usually metachronous $(22,55-64)$ and appears 2-5 years following the eventual chemotherapy and radiotherapy (54), while synchronous breast cancer and lympho- proliferative diseases are much rarer $(18,37,55,58,60,63$, 65). Several case reports describe the coexistence between breast cancer and lymphoma more frequently than expected, with no evidence that the second tumor was treatmentinduced (60-65).

Breast cancer and sarcoma. The first case report of MPNMs including breast cancer and sarcoma was reported in 1936 (66). Another five case reports of these MPNMs have been presented in the literature $(34,67-70)$.

It was Warren who first highlighted how radiotherapy treatment for breast cancer was a risk factor for the development of sarcoma (66).

At the same time, it is important to remember that radiosensitivity is an intrinsic characteristic of the cells of the mammary gland, so that the radiotherapy treatment of a rhabdomyosarcoma of the upper arm can itself be a risk factor for breast cancer development (68).

Approximately $60 \%$ of cancer patients receive radiation therapy during the course of the disease (71) and even after 10 years from radiotherapy a second tumor may develop (72). The definition of radio-induced sarcoma is characterized by the following criteria proposed by Cahan (73) and then modified by Arlen (74): (i) radiant treatment at least three years before developing sarcoma, (ii) onset in the context of previously irradiated tissues, (iii) different histology compared to previously irradiated primary tumor.

Since most patients with breast cancer receive radiation therapy, it is essential to early diagnose this form of sarcoma.

Breast cancer and lung cancer. If a lung lesion is documented in a patient diagnosed with breast cancer, it is always necessary to exclude a lung metastasis, which represents the second most frequent site of metastasis of breast cancer after the bone (75).

However, it is not uncommon to find coexistence of a lung and breast cancer as primitive tumors in the same patient (11, $20,28,32,34,40,48,61,64,76-79)$. This association can be explained mainly considering that: i) breast cancer is the most frequent malignancy in women worldwide (80); ii) in the last years, the mortality of women with an early diagnosis of breast cancer has been significantly reduced and their longer overall survival increases the risk of developing a second tumor (81); iii) lung cancer is the second most common primary malignancy which may develop in patients who have a history of a previous breast malignancy (82).

Breast cancer and gynecological tumors. A strong association between breast cancer and gynecological tumors, especially ovarian cancer, exists, as they are (62, 83-85) influenced by similar risk factors such as early menarchy, low number of pregnancies/nulliparity, obesity as well as high fat diet and fat intake $>38 \%$ of daily calories. 
Table I. Summary of the previous studies describing multiple primary malignant neoplasms.

\begin{tabular}{|c|c|c|c|c|c|c|c|c|}
\hline Year & Author & 1st Tumor & 2nd Tumor & 3rd Tumor & 4th Tumor & 5th Tumor & 6th Tumor & $\begin{array}{l}\text { Synchronous or } \\
\text { Metachronous } \\
\text { primary cancers }\end{array}$ \\
\hline 1980 & Tsuneyoshi M. & Breast carcinoma & Sarcoma & & & & & Metachronous \\
\hline 1984 & Deppe G. & Breast carcinoma & $\begin{array}{c}\text { Colon } \\
\text { adenocarcinoma }\end{array}$ & $\begin{array}{l}\text { Vulva squamous } \\
\text { cell carcinoma }\end{array}$ & & & & Synchronous \\
\hline 1985 & Sugiura K. & Breast carcinoma & $\begin{array}{l}\text { Acute monocytic } \\
\text { leukemia }\end{array}$ & & & & & Metachronous \\
\hline 1985 & Bartal A.H. & Breast carcinoma & Melanoma & & & & & Metachronous \\
\hline 1985 & Patel S. & Breast carcinoma & Lung tumor & Gastric cancer & & & & Synchronous \\
\hline 1986 & Ogasawara K. & Breast carcinoma & $\begin{array}{l}\text { Breast } \\
\text { carcinoma }\end{array}$ & $\begin{array}{c}\text { Lung } \\
\text { carcinoma }\end{array}$ & $\begin{array}{l}\text { Nervous system } \\
\text { tumors (Brain } \\
\text { glioblastoma) }\end{array}$ & $\begin{array}{l}\text { Thyroid } \\
\text { carcinoma }\end{array}$ & & Synchronous \\
\hline 1988 & Shinjo K. & $\begin{array}{c}\text { Rhabdo- } \\
\text { myosarcoma }\end{array}$ & $\begin{array}{c}\text { Breast } \\
\text { carcinoma }\end{array}$ & & & & & Metachronous \\
\hline 1989 & Kobayashi R. & Lymphoma & Breast carcinoma & & & & & Metachronous \\
\hline 1990 & Petru G. & Breast carcinoma & Melanoma & Gastric cancer & & & & Metachronous \\
\hline 1990 & Stierer M. & Breast carcinoma & Lymphoma & & & & & Synchronous \\
\hline 1990 & Newbould MJ. & Breast carcinoma & $\begin{array}{l}\text { Nervous system } \\
\text { tumors } \\
\text { (Peripheral nerves } \\
\text { heath tumour) }\end{array}$ & & & & & Metachronous \\
\hline 1990 & Kohno A. & $\begin{array}{l}\text { B Cell malignant } \\
\text { lymphoma }\end{array}$ & Breast carcinoma & & & & & Metachronous \\
\hline 1991 & Solan M.J. & Breast carcinoma & $\begin{array}{l}\text { Thyroid } \\
\text { carcinoma }\end{array}$ & $\begin{array}{l}\text { Basal cell } \\
\text { carcinoma } \\
\text { (multiple) }\end{array}$ & & & & Metachronous \\
\hline 1992 & Lian D.L. & Cervix tumor & $\begin{array}{l}\text { Larynx squamous } \\
\text { cell carcinoma }\end{array}$ & $\begin{array}{l}\text { Skin squamous } \\
\text { cell carcinoma }\end{array}$ & $\begin{array}{c}\text { Breast } \\
\text { carcinoma }\end{array}$ & $\begin{array}{l}\text { Bladder } \\
\text { tumor }\end{array}$ & $\begin{array}{l}\text { Lung } \\
\text { tumor }\end{array}$ & Metachronous \\
\hline 1992 & Marcos Sanchez F. & $\begin{array}{c}\text { Colon } \\
\text { carcinoma }\end{array}$ & $\begin{array}{c}\text { Renal } \\
\text { carcinoma }\end{array}$ & $\begin{array}{c}\text { Breast } \\
\text { carcinoma }\end{array}$ & - & - & & Metachronous \\
\hline 1994 & Frey B.M. & Breast carcinoma & Lymphoma & & & & & Synchronous \\
\hline 1994 & Bumpers H.L. & $\begin{array}{l}\text { Larynx squamous } \\
\text { cell carcinoma }\end{array}$ & $\begin{array}{l}\text { Lung squamous } \\
\text { carcinoma }\end{array}$ & $\begin{array}{c}\text { Breast } \\
\text { adenocarcinoma }\end{array}$ & $\begin{array}{c}\text { Colon } \\
\text { adenocarcinoma }\end{array}$ & - & & Metachronous \\
\hline 1996 & Padmore R.F. & Breast carcinoma & Melanoma & & & & & Metachronous \\
\hline 1997 & Potzsch C. & $\begin{array}{l}\text { Breast carcinoma } \\
\text { (Synchronous) }\end{array}$ & $\begin{array}{l}\text { Lung carcinoma } \\
\text { (Synchronous) }\end{array}$ & $\begin{array}{c}\text { Renal cell } \\
\text { carcinoma } \\
\text { (Metachronous) }\end{array}$ & $\begin{array}{c}\text { Acute } \\
\text { myelomonocytic } \\
\text { leukemia } \\
\text { (Metachronous) }\end{array}$ & - & & $\begin{array}{l}\text { Synchronous } \\
\text { (Metachronous) }\end{array}$ \\
\hline 2001 & Shousha S. & Breast carcinoma & $\begin{array}{c}\text { Squamous } \\
\text { cell carcinoma } \\
\text { of the oesophagus }\end{array}$ & & & & & Metachronous \\
\hline 2003 & Eros N. & Lymphoma NH & Breast carcinoma & $\begin{array}{c}\text { Basocellular } \\
\text { carcinoma (skin) }\end{array}$ & & & & Metachronous \\
\hline 2003 & Canda M.S. & Breast carcinoma & $\begin{array}{l}\text { Nervous system } \\
\text { tumors (Brain } \\
\text { glioblastoma) }\end{array}$ & & & & & Synchronous \\
\hline 2003 & Roy S.D. & Breast carcinoma & $\begin{array}{l}\text { Axillary mantle } \\
\text { cell lymphoma }\end{array}$ & & & & & Synchronous \\
\hline 2005 & Otrock Z.K. & $\begin{array}{l}\text { Lung adeno- } \\
\text { squamous cell } \\
\text { carcinoma }\end{array}$ & $\begin{array}{l}\text { Bladder } \\
\text { carcinoma }\end{array}$ & $\begin{array}{c}\text { Prostate } \\
\text { adenocarcinoma }\end{array}$ & $\begin{array}{c}\text { Breast } \\
\text { carcinoma }\end{array}$ & & & $\begin{array}{l}\text { Synchronous } \\
\text { metachronous }\end{array}$ \\
\hline 2006 & Fruscalzo A. & Breast carcinoma & $\begin{array}{l}\text { Leukemia } \\
\text { lymphatic } \\
\text { chronic }\end{array}$ & $\begin{array}{l}\text { Ovarian } \\
\text { carcinoma }\end{array}$ & $\begin{array}{c}\text { Endocervical } \\
\text { adenocarcinoma }\end{array}$ & & & Metachronous \\
\hline 2006 & Filippakis G.M. & Teratoma Ovaric & Breast carcinoma & & & & & Synchronous \\
\hline 2006 & Kościuch J. & Breast carcinoma & Gastric carcinoma & & & & & Synchronous \\
\hline 2007 & Agrawal R. & Breast carcinoma & $\begin{array}{l}\text { Cervix squamous } \\
\text { cell carcinoma }\end{array}$ & & & & & Synchronous \\
\hline
\end{tabular}


Table I. Continued

\begin{tabular}{|c|c|c|c|c|c|c|c|c|}
\hline Year & Author & 1st Tumor & 2nd Tumor & 3rd Tumor & 4th Tumor & 5th Tumor & 6th Tumor & $\begin{array}{l}\text { Synchronous or } \\
\text { Metachronous } \\
\text { primary cancers }\end{array}$ \\
\hline 2008 & Cercato MC. & $\begin{array}{l}\text { Breast carcinoma } \\
\text { (Synchronous) }\end{array}$ & $\begin{array}{l}\text { Squamous } \\
\text { cell carcinoma } \\
\text { of the larynx } \\
\text { (Synchronous) }\end{array}$ & $\begin{array}{c}\text { Kidney } \\
\text { carcinoma } \\
\text { (metachronous) }\end{array}$ & $\begin{array}{l}\text { Colon adeno- } \\
\text { carcinoma } \\
\text { (metachronous) }\end{array}$ & & & $\begin{array}{l}\text { Synchronous } \\
\text { metachronous }\end{array}$ \\
\hline 2008 & Izawa N. & Osteosarcoma & Breast cancer & $\begin{array}{l}\text { Colon adeno- } \\
\text { carcinoma }\end{array}$ & Histiocytoma & $\begin{array}{l}\text { Lung } \\
\text { cancer }\end{array}$ & & Metachronous \\
\hline 2012 & Ahmed H. & Breast carcinoma & Osteosarcoma & & & & & Synchronous \\
\hline 2012 & Ko K. & Breast carcinoma & Lung cancer & & & & & Synchronous \\
\hline 2012 & Anania G. & Breast carcinoma & $\begin{array}{l}\text { Caecum adeno- } \\
\text { carcinoma }\end{array}$ & $\begin{array}{l}\text { Sigma adeno- } \\
\text { carcinoma }\end{array}$ & & & & Synchronous \\
\hline 2013 & Băltătescu G.I. & Breast carcinoma & $\begin{array}{c}\text { Ovarian } \\
\text { dysgerminoma }\end{array}$ & & & & & Synchronous \\
\hline 2013 & Sini G. & $\begin{array}{l}\text { Breast carcinoma } \\
\quad \text { (bilateral) }\end{array}$ & $\begin{array}{l}\text { Nervous } \\
\text { system tumors } \\
\text { (Meningioma) }\end{array}$ & $\begin{array}{l}\text { Clear cell } \\
\text { renal cell } \\
\text { carcinoma }\end{array}$ & Melanoma & & & Metachronous \\
\hline 2013 & Kurul S. & Breast carcinoma & Melanoma & Lung cancer & & & & Synchronous \\
\hline 2013 & Pogal J. & Breast carcinoma & $\begin{array}{l}\text { Gallbladder } \\
\text { carcinoma }\end{array}$ & & - & - & & Synchronous \\
\hline 2013 & Higgins L. & Breast carcinoma & $\begin{array}{l}\text { Colon aden- } \\
\text { ocarcinoma }\end{array}$ & & & & & Synchronous \\
\hline 2013 & Pastore A.L. & $\begin{array}{l}\text { Skin squamous } \\
\text { cell carcinoma }\end{array}$ & $\begin{array}{c}\text { Breast } \\
\text { carcinoma }\end{array}$ & $\begin{array}{c}\text { Bladder } \\
\text { carcinoma }\end{array}$ & - & - & & Synchronous \\
\hline 2014 & Karthikeyan V.S. & Breast carcinoma & Gastric cancer & & & & & Metachronous \\
\hline 2014 & Sakellakis M. & Breast carcinoma & Ureter carcinoma & Endometrial & & & & Synchronous \\
\hline 2014 & Powell G. & Osteosarcoma & Breast carcinoma & cancer & & & & Synchronous \\
\hline 2014 & Nishikawa A. & $\begin{array}{l}\text { Breast carcinoma } \\
\text { (accessory } \\
\text { mammary gland) }\end{array}$ & $\begin{array}{l}\text { Lymphoma of } \\
\text { the breast }\end{array}$ & $\begin{array}{l}\text { Rectal adeno- } \\
\text { carcinoma }\end{array}$ & & & & Synchronous \\
\hline 2014 & Yabe N. & Breast carcinoma & Bladder cancer & & & & & Synchronous \\
\hline 2014 & Liu L. & Thyroid carcinoma & Breast carcinoma & & - & - & & Synchronous \\
\hline 2014 & Rastogi M. & $\begin{array}{l}\text { Esophagus } \\
\text { carcinoma }\end{array}$ & $\begin{array}{c}\text { Breast } \\
\text { carcinoma }\end{array}$ & $\begin{array}{l}\text { Base tongue } \\
\text { carcinoma }\end{array}$ & - & - & & Metachronous \\
\hline 2014 & Jafferbhoy S. & Breast carcinoma & $\begin{array}{l}\text { Colon adeno- } \\
\text { carcinoma }\end{array}$ & GIST & & & & Synchronous \\
\hline 2015 & Zhao J. & Colon carcinoma & $\begin{array}{c}\text { Endometrium } \\
\text { cancer }\end{array}$ & $\begin{array}{c}\text { Breast } \\
\text { carcinoma }\end{array}$ & & & & Metachronous \\
\hline 2015 & McPherson J.R. & Breast carcinoma & $\begin{array}{l}\text { Nervous system } \\
\text { tumors (Malignant } \\
\text { peripheral nerve } \\
\text { sheath tumor) }\end{array}$ & Neurofibroma & & & & Metachronous \\
\hline 2015 & Klairmont M. & Breast carcinoma & Lung cancer & $\begin{array}{l}\text { Colon adeno- } \\
\text { carcinoma }\end{array}$ & Esophagus cancer & & & Synchronous \\
\hline 2015 & Williamson C.W. & Breast carcinoma & Giant cell tumor; & Lymphoma; & Melanoma & & & Synchronous \\
\hline 2015 & Tian F. & Breast carcinoma & $\begin{array}{l}\text { Hepatocellular } \\
\text { carcinoma }\end{array}$ & & & & & Synchronous \\
\hline 2016 & Lopez J.J. & Breast carcinoma & $\begin{array}{l}\text { Tricholemmal } \\
\text { tumor }\end{array}$ & & & & & Synchronous \\
\hline 2016 & Vennepureddy A. & Breast cancer & Myeloma & $\begin{array}{l}\text { Monoclonal B-Cell } \\
\text { lymphocytosis }\end{array}$ & & & & Synchronous \\
\hline 2016 & Cohen P.R. & Breast cancer & $\begin{array}{l}\text { Hodgkin } \\
\text { lymphoma }\end{array}$ & $\begin{array}{l}\text { Lung } \\
\text { cancer }\end{array}$ & $\begin{array}{l}\text { Kidney } \\
\text { cancer }\end{array}$ & $\begin{array}{l}\text { Thyroid } \\
\text { cancer }\end{array}$ & & Metachronous \\
\hline 2016 & Ari A. & Breast carcinoma & & $\begin{array}{l}\text { Colon adeno- } \\
\text { carcinoma }\end{array}$ & & & & Synchronous \\
\hline 2017 & Liu J. & Breast carcinoma & $\begin{array}{l}\text { Mantle Cell } \\
\text { Lymphoma } \\
\text { (MCL) }\end{array}$ & & & & & Synchronous \\
\hline
\end{tabular}


Table I. Continued

\begin{tabular}{|c|c|c|c|c|c|c|c|}
\hline Year & Author & 1st Tumor & 2nd Tumor & 3rd Tumor & 4th Tumor & 5th Tumor & $\begin{array}{l}\text { Synchronous or } \\
\text { Metachronous } \\
\text { primary cancers }\end{array}$ \\
\hline 2017 & Shuayb M.D. & Breast carcinoma & $\begin{array}{l}\text { Nervous system } \\
\text { tumors (malignant } \\
\text { peripheral nerve } \\
\text { sheath tumors } \\
\text { of the breast) }\end{array}$ & & & & Synchronous \\
\hline 2017 & Alharbi A. & Breast carcinoma & $\begin{array}{l}\text { Appendicular } \\
\text { cancers }\end{array}$ & & & & Synchronous \\
\hline 2017 & Nyqvist J. & $\begin{array}{l}\text { Endometrium } \\
\text { adenocarcinoma } \\
\text { (Metachronous) }\end{array}$ & $\begin{array}{c}\text { Colon } \\
\text { adenocarcinoma } \\
\text { (Metachronous) }\end{array}$ & $\begin{array}{c}\text { Melanoma } \\
\text { (Synchronous) }\end{array}$ & $\begin{array}{c}\text { Breast } \\
\text { carcinoma } \\
\text { (Synchronous) }\end{array}$ & $\begin{array}{c}\text { Sarcoma } \\
\text { (Synchronous) }\end{array}$ & $\begin{array}{c}\text { Metachronous } \\
\text { synchronous }\end{array}$ \\
\hline 2018 & Marković M. & Breast carcinoma & $\begin{array}{l}\text { Colon adeno- } \\
\text { carcinoma }\end{array}$ & GIST & & & Metachronous \\
\hline 2018 & Ágoston E.I. & Breast carcinoma & $\begin{array}{l}\text { Colon adeno- } \\
\text { carcinoma }\end{array}$ & Renal cell carcinoma & & & Synchronous \\
\hline 2018 & Sapalidis K. & $\begin{array}{c}\text { Tonsillar oral } \\
\text { cancer breast } \\
\text { cancer }\end{array}$ & Lung cancer & Breast carcinoma & & & Metachronous \\
\hline 2018 & Martin M. & Uter cancer & Lung carcinoid & Breast carcinoma & Melanoma & & Metachronous \\
\hline 2018 & Parekh J.D. & Breast cancer & $\begin{array}{c}\text { Rectal } \\
\text { squamous cell }\end{array}$ & $\begin{array}{l}\text { Renal cell } \\
\text { carcinomas }\end{array}$ & & & Metachronous \\
\hline 2018 & Jin B. & Breast cancer & Lung cancer & & & & Synchronous \\
\hline
\end{tabular}

Women with sporadic breast cancer have a high risk of developing sporadic ovarian cancer, and this risk increases if we consider genetic syndromes such as the inherited BRCA1 and BRCA2 gene mutations $(8,86,87)$ and the Lynch syndrome type II, which also includes colon, endometrium and high gastrointestinal tract tumors (87).

In hereditary tumor syndromes a single germline mutation in a single gene leads to a high risk of cancer (87). The association between primary breast and ovarian tumors is very strong and so in the oncological follow-up of breast cancer, the ovaries are always investigated to rule out an ovarian lesion. In fact, unlike breast cancer, ovarian cancer is less frequent but is characterized by poor prognosis, considering that the overall 5-year survival is $50 \%$ and in stages III and IV it drops to $15-20 \%$ (88).

Breast cancer and thyroid cancer. Women with a history of thyroid cancer have a greater risk of developing a second tumor when compared to the normal population, especially breast cancer $(89,90)$. In the case of multiple tumors, the second most frequent site is the breast gland (90) and the risk is higher if thyroid cancer appears in women aged less than 50 years (91).

These two tumors share numerous molecular mechanisms that might activate the carcinogenic process. Among these mechanisms, mutations in the thyroid receptor play an important role in breast hyperplasia (92).

Finally, there are genetic syndromes such as the Cowden syndrome or the Cowden-like syndrome, in which breast and thyroid cancers are associated (93). It is therefore not surprising that there are several case reports of breast and thyroid MPMNs, both synchronous $(48,94)$ and metachronous $(10,64)$.

Breast cancer and urological tumors. Bladder and breast cancers share extrinsic risk factors (including personal lifestyle, ultraviolet radiation, smoking and alcohol) and some genetic mutations in signal transduction pathways $(95,96)$. Damrauer et al. showed that basal-like and luminal-like subtypes of bladder cancer surprisingly reflect the hallmarks of breast biology (97).

If the generic association of breast cancer with urological tumors can be considered sporadic $(11,12,16,31,44,61$, $76,86,95,96)$, the same cannot be said for bladder cancer $(11,12,76,95)$.

\section{Discussion}

Between 1980 and 2018 only 12 cases of MPMNs involving the breast gland or the skin have been described (10-21). Among these, only two $(11,12)$ cases report the coexistence of breast and cutaneous squamous cell cancers (Table I). The first case was described by Lian in 1992 (11), who focused on a patient suffering by six metachronous primary malignant neoplasms in a period of 10 years (cervix, larynx, skin, left breast, bladder and right lung) (11).

The second study described by Pastore et al. in 2013 (12) reported the clinical case of a male patient who developed 
synchronous carcinoma of the bladder, squamous cell carcinoma of the forehead skin, and breast cancer (12).

We report the case of a patient who developed two histologically distinct malignancies, meeting the inclusion criteria of Warren and Gates to be defined as a MPMN (3). The immunohistochemical analyses, carried out on the two surgical specimens, showed the different primitiveness of the lesions, excluding a metastatic origin for one of them: a wide squamous cell carcinoma of the pectoral skin and an invasive ductal carcinoma of the breast. These can be considered as two synchronous and non-metachronous tumors since the diagnosis was performed within 6 months of each other (3).

When Billroth (1) in 1889 diagnosed for the first time a case of MPMN, the phenomenon of multiple tumors was still rare. Nowadays, the diagnosis of second primary neoplasms is not so infrequent. To our knowledge, the case that we describe is the second, after the one described by Pastore (12) reporting a synchronism between giant squamous cell carcinoma of the right breast skin and ipsilateral breast cancer. Synchronous tumors are much rarer and often attributed to known and unknown genetic predispositions (19).

In our case, we excluded any syndromic frameworks, such as Li Fraumeni (TP53) or Von Hippel Lindau syndrome, or genetic mutations (i.e. BRCA1/2), that might have predisposed to the development of multiple tumors. Moreover, despite the fact that germline TP53 mutations are rare in patients with primary tumors, it was not possible to exclude the possibility that a sporadic mutation in TP53 may have led to a predisposition to multiple tumors (98-100).

Our patient did not have a family history of malignancies, which led us to verify the presence of intrinsic and extrinsic risk factors that could justify the sporadic occurrence of MPMNs.

According to the literature, principal risk factors known for MPMNs are tobacco, alcohol or exposure to radiation (101). We excluded the first two factors, but we could not exclude a previous prolonged exposure to sun light. Our patient presented a Fitzpatrick skin phototype II with a higher possibility to develop pre-neoplastic lesions such as actinic keratosis (AK).

$\mathrm{AK}$ is a lesion that arises as a result of excessive exposure to solar radiation and is highly frequent in Fitzpatrick skin phototype I and II (102). AK typically occurs in areas principally exposed to the sun light (face, upper and lower limbs) and may often evolve into a squamous cell carcinoma.

Our patient did not present any $\mathrm{AK}$ at the physical examination but we could not rule out that an AK may have been present before the squamous cell carcinoma.

In addition to sunlight, other non-sun related risk factors, such as exposition to insecticides, herbicides, and fungicides lead to the development of squamous cell carcinoma of the skin (101). Our patient was a housewife living in an urban context and hardly ever came into contact with non-sun risk factors.
Age is a risk factor for the development of MPMNs (9). Considering that longevity is increasing in the general population, a higher incidence of MPMNs is expected, particularly in patients with a positive history of malignancy (103). However, our patient did not have a personal history of previous tumors.

We cannot therefore attribute it with certainty to a known genetic syndrome or a sporadic occurrence justified by known risk factors. The difficulty in deciding the best therapeutic strategy has led us to review all the MPMNs case reports including breast cancer.

Whatever the cause and/or the established risk factors, some parameters need to be considered for the treatment of MPMNs: the stages of the different neoplasms, tumor behavior, patient's age, life expectancy, and comorbidities.

While in metachronous tumors the therapeutic course always involves the sequential treatment of each single tumor, in synchronous tumors we found that a personalized and singular treatment is decided after an appropriate evaluation in multidisciplinary team meetings and a consensus on a therapeutic strategy (104), as it is done in the case of an inflammatory breast cancer (105). If in localized cancer it is possible to choose between surgical or chemo/radio-therapy approach to treat coexisted tumors, in advanced cancer disease the antitumor therapy selection is difficult and often not supported by research evidence (104).

In our case, the multidisciplinary team decided to start with neoadjuvant chemotherapy to down-stage the two tumors, and to reach a better local control of the disease. Definitive histopathologic examination showed that chemotherapy had successfully down staged both diseases and, in addition to surgery, made possible a full recovery.

To our knowledge, our case is the first in the literature reporting simultaneous and complete pathological response of two different tumors using a single chemotherapy line protocol composed by carboplatin and taxane.

\section{Conclusion}

Nowadays, multiple primary cancers are not rare entities. Improved cancer care and increased survival have led to an increase in the number of patients developing multiple cancers.

While it is possible to eliminate risk factors such as smoking, alcohol consumption and unhealthy lifestyle, notmodifiable risk factors such as the genetic susceptibility, the state of immune deficiencies and the sometimes-irreversible effects of anticancer therapies cannot be managed.

Although no familial history or carcinogenetic factor could be evidenced, the occurrence of multiple tumors, especially the synchronous ones, is unlikely to be fortuitous.

Patients who develop multiple cancers may not fall into one of the hereditary syndromes, but it is possible that there are unknown and/or rare genetic predispositions that 
determine the development of multiple tumors. For this reason, all multiple cancer patients should be followed-up for the development of subsequent cancers.

A complete pre-operative evaluation and interdisciplinary collaboration can always guarantee the best possible treatment for a patient.

Nowadays, the description of case reports is of fundamental importance since they strongly contribute to the progress of medical knowledge, generating new hypotheses and acting as stimulus for the scientific community to verify them. This study portrays a particularly rare clinical picture and provides first evidence of efficacy of a therapeutic protocol that deserves to be investigated in more detail.

\section{Conflicts of Interest}

The Authors have no conflicts of interest to declare regarding this study.

\section{Authors' Contributions}

A.D.L. collected the data, conceived and wrote the paper; F.F. collected the data; M.V. collected the data; R.C. reviewed the paper; G.L.T. reviewed the paper; L.B. collected the data; M.M. reviewed the paper; M.I.A. collected the data, conceived and wrote the paper

\section{References}

1 Billroth T: Die Allgemeine chirurgische pathologie und therapie. In: G.Reimer (ed.). 51 Vorlesungen-Ein Handbuch fur Studierende und Artze, 14. Auflage, Berlin, pp. 908, 1889.

2 Moertel CG, Dockerty MB and Baggenstoss AH: Multiple primary malignant neoplasms. II. Tumors of different tissues or organs. Cancer 14: 231-237, 1961. PMID: 13771653.

3 Warren S: Multiple malignant tumors. A survey of the literature and statistical study. Am J Cancer 16: 1358-1414, 1932.

4 Demandante CG, Troyer DA and Miles TP: Multiple primary malignant neoplasms: case report and a comprehensive review of the literature. Am J Clin Oncol 26: 79-83, 2003. PMID: 12576929.

$5 \mathrm{Xu} \mathrm{LL}$ and $\mathrm{Gu} \mathrm{KS}$ : Clinical retrospective analysis of cases with multiple primary malignant neoplasms. Genet Mol Res 13: 92719284, 2014. PMID: 24682981. DOI: 10.4238/2014. March.12.19

6 Group AW: Italian cancer figures, report 2013: multiple tumours. Epidemiol Prev 37: 1-152, 2013. PMID: 24259384.

7 Yamamoto S, Yoshimura K, Ri S, Fujita S, Akasu T and Moriya Y: The risk of multiple primary malignancies with colorectal carcinoma. Dis Colon Rectum 49: S30-S36, 2006. PMID: 17106813. DOI: 10.1007/s10350-006-0600-8

8 Varol U, Kucukzeybek Y, Alacacioglu A, Somali I, Altun Z, Aktas S and Oktay Tarhan M: BRCA genes: BRCA 1 and BRCA 2. J BUON 23: 862-866, 2018. PMID: 30358186.

9 Spratt JS and Hoag MG: Incidence of multiple primary cancers per man-year of follow up: 20-year review from the Ellis Fischel State Cancer Hospital. Ann Surg 164: 775-784, 1966. PMID: 5923101. DOI: 10.1097/00000658-196611000-00001

10 Solan MJ: Multiple primary carcinomas as sequelae of treatment of pulmonary tuberculosis with repeated induced pneumothoraces. Case report and review of the literature. Am J Clin Oncol 14: 49-51, 1991. PMID: 1987738.
11 Lian DL, Hsu CP, Chen CY, Chen CL, Lin CT and Wang PY. Multiple primary malignant neoplasms: a case report. Zhonghua Yi Xue Za Zhi (Taipei) 50: 504-508, 1992. PMID: 1338029.

12 Pastore AL, Palleschi G, Autieri D, Leto A, Ripoli A, Maggioni C, Moschese D, Al Salhi Y, Porta N, Di Cristofano C, Fuschi A, Silvestri L, Della Rocca C, Tomao S, Petrozza V and Carbone A: Synchronous primary neoplasms of the bladder, skin and breast in a male patient: a case report. World J Surg Oncol 11: 282, 2013. PMID: 24139546. DOI: 10.1186/1477-7819-11-282

13 Bartal AH and Pinsky CM: Malignant melanoma appearing in a post-mastectomy lymphedematous arm: a novel association of double primary tumors. J Surg Oncol 30: 16-18, 1985. PMID: 4079413.

14 Petru G, Fuhrmann W and Borkenstein J: Occurrence of 3 primary carcinomas within 26 months. Wien Med Wochenschr 140: 212-213, 1990. PMID: 2163572.

15 Padmore RF, Lara JF, Ackerman DJ, Gales T, Sigurdson ER, Ehya H, Cooper HS and Patchefsky AS: Primary combined malignant melanoma and ductal carcinoma of the breast. A report of two cases. Cancer 78: 2515-2525, 1996. PMID: 8952560.

16 Sini G, Colombino M, Lissia A, Maxia S, Gulino M, Paliogiannis P, Palomba G, Palmieri G, Cossu A and Rubino C: Primary dermal melanoma in a patient with a history of multiple malignancies: a case report with molecular characterization. Case Rep Dermatol 5: 192-197, 2013. PMID: 23904845. DOI: $10.1159 / 000354032$

17 Kurul S, Akgun Z, Saglam EK, Basaran M, Yucel S and Tuzlali S: Successful treatment of triple primary tumor. Int J Surg Case Rep 4: 1013-1016, 2013. PMID: 24091078. DOI: 10.1016/ j.ijscr. 2013.08.010

18 Williamson CW, Paravati A, Ghassemi M, Lethert K, Hua P, Hartman $\mathrm{P}$ and Sanghvi P: Five simultaneous primary tumors in a single patient: a case report and review of the literature. Case Rep Oncol 8: 432-438, 2015. PMID: 26600775. DOI: $10.1159 / 000440799$

19 Nyqvist J, Persson F, Parris TZ, Helou K, Kenne Sarenmalm E, Einbeigi Z, Borg $\AA$, Karlsson $\mathrm{P}$ and Kovács A: Metachronous and synchronous occurrence of 5 primary malignancies in a female patient between 1997 and 2013: a case report with germline and somatic genetic analysis. Case Rep Oncol 10: 1006-1012, 2017. PMID: 29279706. DOI: $10.1159 / 000484403$

20 Martin, Joshua K. Sabari, Gulisa Turashvili, Darragh F. Halpenny, Hira Rizvi, Natalie Shapnik and Vicky Makkera: Next-generation sequencing based detection of germline and somatic alterations in a patient with four metachronous primary tumors. Gynecol Oncol Rep 24: 94-98, 2018. PMID: 29915805. DOI: $10.1016 /$ j.gore.2018.04.004

21 Eros N, Karolyi Z, Kovács A, Matolcsy A, Barna T and Kelényi G: Large B-cell lymphoma of the leg in a patient with multiple malignant tumours. Acta Derm Venereol 83: 354-357, 2003. PMID: 14609103. DOI: 10.1080/00015550310010612

22 Koh HK, Sober AJ and Carey RA: Possible association between malignant melanoma and breast cancer. Arch Dermatol 123: 712-713, 1987. PMID: 3579352.

23. Pogal, Vijay Kumar, Nuzhat Husain, and Sanjeev Misra: A unique case of two primaries: carcinoma breast with carcinoma gallbladder. BMJ Case Rep 2013: bcr2013009243, 2013. PMID: 23715835. DOI: 10.1136/bcr-2013-009243 
24 Tian F, Cui X, Li L, Lu H, Rong W, Bi C and Wu J: Synchronous primary breast cancer and hepatocellular carcinoma in a male patient: a case report. Int J Clin Exp Pathol 8: 11722-11728, 2015. PMID: 26617917.

25 Rastogi M, Singh S, Singh S, Gupta S and Dwivedi RC: Triple primary malignant neoplasms including breast, esophagus and base tongue in an elderly male: a case report. J Cancer Res Ther 10: 1109-1111, 2014. PMID: 25579565. DOI: 10.4103/09731482.144639

26 Kościuch J, Zielonka TM, Ziółkowski B, Domagała-Kulawik J, Bogacka-Zatorska E and Chazan R: Breast carcinoma and anaplastic gastric carcinoma with pleural metastases in patient after mastectomy 30 years ago. Pneumonol Alergol Pol 74: 129131, 2006. PMID: 17175993

27 Karthikeyan VS, Sistla SC, Srinivasan R, Basu D, Panicker LC, Ali SM and Rajkumar N: Metachronous multiple primary malignant neoplasms of the stomach and the breast: Report of two cases with review of literature. Int Surg 99: 52-55, 2014. PMID: 24444270. DOI: 10.9738/INTSURG-D-13-00056.1

28 Patel S, Alfonso AE, Landis J and Suarez J: Three synchronous multiorgan primary cancers. All stage I. Arch Surg 120: 11821184, 1985. PMID: 4038063

29 Shousha S, Fawcett A, Luqmani YA and Theodorou N: Multifocal squamous cell carcinoma of the oesophagus following radiotherapy for bilateral breast carcinoma. J Clin Pathol 54: 718-720, 2001. PMID: 11533082. DOI: $10.1136 /$ jcp.54.9.718

30 Deppe G, Dolan TE, Zbella EA and Heredia R: Synchronous multiple primary malignant neoplasms of the breast, colon and vulva. A case report. J Reprod Med 29: 878-880, 1984. PMID: 6097686

31 Marcos Sánchez F, Salvador Fernández M, Juárez Ucelay F and Druet Ampuero J: Carcinomas of the colon, kidney, and breast in the same patient. A new case of multiple primary malignant neoplasms. An Med Interna 9: 257, 1992. PMID: 1324018.

32 Bumpers HL, Natesha RK, Barnwell SP and Hoover EL: Multiple and distinct primary cancers: a case report. J Natl Med Assoc 86: 387-388, 1994. PMID: 8046769.

33 Cercato MC, Colella E, Ferraresi V, Diodoro MG and Tonachella R: Report of two cases of quintuple primary malignancies and review of the literature. Anticancer Res 28: 2953-2958, 2008. PMID: 19031939.

34 Izawa N, Matsumoto S, Manabe J, Tanizawa T, Hoshi M, Shigemitsu T, Machinami R, Kanda H, Takeuchi K, Miki Y, Arai M, Shirahama S and Kawaguchi N: A Japanese patient with Li-Fraumeni syndrome who had nine primary malignancies associated with a germline mutation of the p53 tumor-suppressor gene. Int J Clin Oncol 13: 78-82, 2008. PMID: 18307025. DOI: 10.1007/s10147-007-0692-8

35 Anania G, Santini M, Marzetti A, Scagliarini L, Vedana L, Resta G and Cavallesco G: Synchronous primary malignant tumors of the breast, caecum and sigma. Case report. G Chir 33: 409-410, 2012. PMID: 23140927.

36 Higgins L, Robertson I, Khan W and Barry K: Synchronous breast and colon cancer: factors determining treatment strategy. BMJ Case Rep 10: 2013, 2013. PMID: 23845673. DOI: 10.1136/bcr-2013-009450

37 Nishikawa A, Kasai H, Koyama Y, Koide N, Iijima A, Shimojo $\mathrm{H}$ and Kumeda S: Synchronous ipsilateral carcinoma of the accessory mammary gland and primary lymphoma of the breast with subsequent rectal carcinoma: report of a case. World J Surg Oncol 12: 286, 2014. PMID: 25217973. DOI: $10.1186 / 1477-7819-12-286$

38 Jafferbhoy S, Paterson H and Fineron P: Synchronous gist, colon and breast adenocarcinoma with double colonic polyp metastases. Int J Surg Case Rep 5: 523-526, 2014. PMID: 25014550. DOI: $10.1016 /$ j.ijscr.2014.04.020

39 Zhao J, Tan Y, Wu Y, Zhao W, Wu J, Ji M, Shi L, Jiang J and Wu C: A rare case of eight multiple primary malignant neoplasms in a female patient: A case report and review of the literature. Oncol Lett 9: 587-590, 2015. PMID: 25624886. DOI: 10.3892/ol.2014.2789

40 Klairmont M, Kopkash K, Favuzza J, Hill M, Rao R, Mahon B and Seder CW: Four Synchronous Primary Malignancies of the Breast, Lung, Colon and Esophagus. Anticancer Res 35: 61596162, 2015. PMID: 26504043.

41 Ari A, Tatar C, Buyukasik K, Segmen O, Cakir C and Arikan S: Synchronous male breast and colon cancer presenting with ileus: A case report. Int J Surg Case Rep 28: 31-33, 2016. PMID: 27677113. DOI: 10.1016/j.ijscr.2016.09.023

42 Alharbi A, Aboasamh G and Shafik Y: Appendicular and breast cancers in an old lady: a case report. J Surg Case Rep 2017: rjx251, 2017. PMID: 29308181. DOI: $10.1093 /$ jscr/rjx251

43 Marković M, Jurišić V, Petrović M, Dagović A, Stanković V and Mitrović S: Appearance of ductal breast and colon carcinoma with gastrointestinal stromal tumor (GIST) in a female patient: an extremely rare case. Rom $\mathrm{J}$ Morphol Embryol 59: 613-617, 2018. PMID: 30173272.

44 Ágoston EI, Somorácz Á, Madaras L, Zaránd A, Szentmártoni G, Orosz Z, Dank M and Baranyai Z: Successful treatment of three synchronous primary malignant tumours - reflection on surgical, pathological and oncological aspects and decision making. J Surg Case Rep 2018: rjy041, 2018. PMID: 29657704. DOI: $10.1093 /$ jscr/rjy041

45 Bittorf B, Kessler H, Merkel S, Bru"ckl W, Wein A, Ballhausen WG, Hohenberger W and Günther K: Multiple primary malignancies: An epidemiological and pedigree analysis of 57 patients with at least three tumours. Eur J Surg Oncol 27: 302-313, 2001. PMID: 11373110. DOI: 10.1053/ejso.2001.1112

46 Wang $\mathrm{C}$, Zhao $\mathrm{P}$, Bai X, Wang $\mathrm{H}$ and Bai Y: Breast cancer in multiple primary malignant neoplasms, epidemiological and clinical analysis. Zhonghua Yi Xue Za Zhi 82: 1229-1231, 2002. PMID: 12425798

47 Arrangoiz R, Papavasiliou P, Dushkin H and Farma JM: Case report and literature review: Metastatic lobular carcinoma of the breast an unusual presentation. Int J Surg Case Rep 2: 301-305, 2011. PMID: 22096760. DOI: 10.1016/j.ijscr.2011.06.010

48 Ogasawara K, Ogawa A, Shingai J, Kayama T, Wada T, Namiki $\mathrm{T}$ and Suzuki J: Synchronous multiple primary malignant tumors accompanied by glioblastoma. Case report. Neurol Med Chir (Tokyo) 26: 908-912, 1986. PMID: 2437485. DOI: 10.2176/ nmc. 26.908

49 Canda MS, Guray M, Erbayraktar RS, Acar F and Canda T: Association of invasive breast carcinoma and glioblastoma multiforme: a case report with histological and immunohistochemical features. Turk J Med Sci 34: 131-135, 2004.

50 Gavrilovic IT and Posner JB: Brain metastases: epidemiology and physiopathology. J Neurooncol 75: 5-14, 2005. PMID: 16215811. DOI: $10.1007 / \mathrm{s} 11060-004-8093-6$ 
51 Newbould MJ, Wilkinson $\mathrm{N}$ and Mene A: Post-radiation malignant peripheral nerve sheath tumour: a report of two cases. Histopathology 17: 263-265, 1990. PMID: 2242854.

52 McPherson JR, Ong CK, Ng CC, Rajasegaran V, Heng HL, Yu WS, Tan BK, Madhukumar P, Teo MC, Ngeow J, Thike AA, Rozen SG, Tan PH, Lee AS, Teh BT and Yap YS: Wholeexome sequencing of breast cancer, malignant peripheral nerve sheath tumor and neurofibroma from a patient with neurofibromatosis type 1. Cancer Med 4: 1871-1878, 2015. PMID: 26432421. DOI: 10.1002/cam4.551

53 Shuayb $M$ and Begum R: Unusual primary breast cancer malignant peripheral nerve sheath tumor: a case report and review of the literature. J Med Case Rep 11: 161, 2017. PMID: 28622765. DOI: 10.1186/s13256-017-1332-1

54 Harvey EB and Brinton LA: Second cancer following cancer of the breast in Connecticut, 1935-82. Natl Cancer Inst Monogr 68: 99-112, 1985. PMID: 4088315.

55 Dutta Roy S, Stafford JA, Scally J and Selvachandran SN: A rare case of breast carcinoma coexisting with axillary mantle cell lymphoma. World J Surg Oncol 1: 1-3, 2003. PMID: 14664721. DOI: $10.1186 / 1477-7819-1-27$

56 Sugiura K, Koyama K, Katayama J, Nonaka K and Yagi Y: A report of five cases of multiple cancer with hematologic malignancies. Gan No Rinsho 31: 963-8, 1985. PMID: 3861885.

57 Kobayashi R, Osada T, Hamaguchi M, Ohi T, Inden Y, Hara K, Tomiyama J, Kitayama J, Tsukui G and Kobori O: A carcinoma of the right breast arising after a mastectomy in primary malignant lymphoma of the left breast. Gan No Rinsho 35: 1077-1080, 1989. PMID: 2549281.

58 Stierer M, Rosen HR, Heinz R and Hanak H: Synchrony of malignant lymphoma and breast cancer. JAMA 263: 2922-2923, 1990. PMID: 2160024.

59 Kohno A, Kohriyama K and Arimori S: Breast cancer and B cell malignant lymphoma associated with Sjögren's syndrome a case report and review of literature in Japan. Ryumachi 30: 388-393, 395; discussion 393-394, 1990. PMID: 2084862.

60 Frey BM, Morant R, Senn HJ, Fisch T and Schmid U: Simultaneous occurrence of breast carcinoma and malignant lymphoma. Case observations and literature review. Schweiz Med Wochenschr 124: 1010-1016, 1994. PMID: 8023099.

61 Potzsch C, Fetscher S, Mertelsmann R and Lubbert M: Acute myelomonocytic leukemia secondary to synchronous carcinomas of the breast and lung, and to metachronous renal cell carcinoma. J Cancer Res Clin Oncol 123: 678-680, 1997. PMID: 9620229.

62 Fruscalzo A, Damante G, Calcagno A, Di Loreto C and Marchesoni D: Four primary malignancies successively occurred in a BRCA2 mutation carrier: a case report. Cancer Invest 24: 611614, 2006. PMID: 16982466. DOI: 10.1080/073579 00600894872

63 Vennepureddy A, Motilal Nehru V, Liu Y, Mohammad F and Atallah JP: Synchronous diagnosis of multiple myeloma, breast cancer, and monoclonal B-cell lymphocytosis on initial presentation. Case Rep Oncol Med 2016: 7953745, 2016. PMID: 27247815. DOI: $10.1155 / 2016 / 7953745$

64 Cohen PR: Segmental neurofibromatosis and cancer: report of triple malignancy in a woman with mosaic Neurofibromatosis 1 and review of neoplasms in segmental neurofibromatosis. Dermatol Online J 22: pii: 13030/qt66k5j4wt, 2016. PMID: 27617721.

65 Liu J, Wei H, Zhu K, Lai L, Han X and Yang Y: Male breast cancer and mantle cell lymphoma in a single patient: A case report and literature review. Medicine (Baltimore) 96(48): e8911, 2017. PMID: 29310379. DOI: 10.1097/MD.00 00000000008911

66 Warren S and Sommer GN: Fibrosarcoma of the soft parts with special reference to recurrence and metastasis. Arch Surg 33: 425-50. 1936. DOI: 10.1001/archsurg.1936.01190030078004

67 Tsuneyoshi M and Enjoji M: Postirradiation sarcoma (malignant fibrous histiocytoma) following breast carcinoma: an ultrastructural study of a case. Cancer 45(6): 1419-1423, 1980. PMID: 6244081.

68 Shinjo K, Asai T, Saito S, Tuboi S and Tamura J: Multiple primary malignant tumors of rhabdomyosarcoma in an upper arm and breast cancer. Gan No Rinsho 34(1): 123-129, 1988. PMID: 3339846.

69 Ahmed H, Salama A, Salem SE and Bahnassy AA: A case of synchronous double primary breast carcinoma and osteosarcoma: Mismatch repair genes mutations as a possible cause for multiple early onset malignant tumors. Am J Case Rep 13: 218-223, 2012. PMID: 23569533. DOI: 10.12659/ AJCR.883382

70 Powell G, Barth L, Todd R and Ganesan R: Primary uterine osteosarcoma presenting synchronously with bilateral breast carcinomas. BMJ Case Rep 2014: pii: bcr2013201502, 2014. PMID: 24898994. DOI: 10.1136/bcr-2013-201502

71 Halperin EC, Perez CA and Brady LW: Perez and Brady's principles and practice of radiation oncology. 5th edition. Philadelphia: Wolters Kluwer/Lippincott Williams \& Wilkins, c2008.

72 Hall EJ and Wuu CS: Radiation-induced second cancers: the impact of 3D-CRT and IMRT. Int J Radiat Oncol Biol Phys 56: 83-88, 2003. PMID: 12694826.

73 Cahan WG and Woodard HQ: Sarcoma arising in irradiated bone; report of 11 cases. Cancer 1: 3-29, 1948. PMID: 18867438.

74 Arlen M, Higinbotham NL, Huvos AG, Marcove RC, Miller T and Shah IC: Radiation-induced sarcoma of bone. Cancer 28: 1087-1099, 1971. PMID: 5288429.

75 Wu SG, Li H, Tang LY, Sun JY, Zhang WW, Li FY, Chen YX and $\mathrm{He} \mathrm{ZY}$ : The effect of distant metastases sites on survival in de novo stage-IV breast cancer: A SEER database analysis. Tumour Biol 39(6): 1010428317705082, 2017. PMID: 286538 87. DOI: $10.1177 / 1010428317705082$

76 Otrock ZK, Mahfouz RA and Salem ZM: Four primary tumors of lung, bladder, prostate, and breast in a male patient. South Med J 98(9): 946-949, 2005. PMID: 16217994. DOI: 10.1097/ 01.smj.0000173086.37625.e2

77 Ko K, Ro JY, Hong EK and Lee S: Micropapillary lung cancer with breast metastasis simulating primary breast cancer due to architectural distortion on images. Korean J Radiol 13(2): 249253, 2012. PMID: 22438695. DOI: 10.3348/kjr.2012.13.2.249

78 Sapalidis K, Schizas N, Lazopoulos A, Kamparoudi P, Paliouras D, Sardeli C, Konstantinou F, Chatzinikolaou F, Sarafis P, Zarogoulidis P, Kougioumtzi I, Katsikogiannis N, Karapantzos I, Karapantzou C, Laskou S, Koulouris C, Mantalobas S, Giannakidis D, Kesisoglou I and Barbetakis N: Multiple metachronous and synchronous malignancies with lung and thorax involvement. Report of two cases. Respir Med Case Rep 24: 5-7, 2018. PMID: 29977745. DOI: 10.1016/j.rmcr.2018.03.006

79 Jin B, Zhang S, Chuang X, Yu P, Chen Y, Teng Y, Che X, Fan Y, Zheng C, Li X, Qiu X, Qu X and Liu Y: Breast cancer and synchronous multiple primary lung adenocarcinomas with 
heterogeneous mutations: a case report. BMC Cancer 18(1): 1138, 2018. PMID: 30453894. DOI: 10.1186/s12885-018-5011-4

80 Siegel RL, Miller KD and Jemal A: Cancer Statistics, 2017. CA Cancer J Clin 67: 7-30, 2017. PMID: 28055103. DOI: 10.3322/ caac. 21387

81 Mariotto AB, Rowland JH, Ries LA, Scoppa S and Feuer EJ: Multiple cancer prevalence: a growing challenge in long- term survivorship. Cancer Epidemiol Biomarkers Prev 16: 566-571, 2007. PMID: 17372253. DOI: 10.1158/1055-9965.EPI-06-0782

82 Liu YY, Chen YM, Yen SH, Tsai CM and Perng RP: Multiple primary malignancies involving lung cancer-clinical characteristics and prognosis. Lung Cancer 35: 189-194, 2002. PMID: 11804692.

83 Filippakis GM, Lagoudianakis EE, Genetzakis M, Antonakis P, Papadima A, Boussiotou A, Katergiannakis V and Manouras A: Squamous cell carcinoma arising in a mature cystic teratoma of the ovary with synchronous invasive lobular breast cancer: case report. Eur J Gynaecol Oncol 27(5): 537-540, 2006. PMID: 17139997.

84 Agrawal R: Synchronous dual malignancy: successfully treated cases. J Cancer Res Ther 3(3): 153-156, 2007. PMID: 18079578.

85 Băltătescu GI, Așchie M and Sârbu V: Synchronous ovarian dysgerminoma and breast carcinoma in a patient with positive immunostain of BRCA1. Chirurgia (Bucur) 108(2): 259-263, 2013. PMID: 23618579.

86 Sakellakis M, Peroukides S, Iconomou G, Boumpoucheropoulos $\mathrm{S}$ and Kalofonos H: Multiple primary malignancies: a report of two cases. Chin J Cancer Res 26(2): 215-218, 2014. PMID: 24826064. DOI: 10.3978/j.issn.1000-9604.2014.02.15

87 Menko FH, Ter Stege JA, van der Kolk LE, Jeanson KN, Schats W, Moha DA and Bleiker EMA: The uptake of presymptomatic genetic testing in hereditary breast-ovarian cancer and Lynch syndrome: a systematic review of the literature and implications for clinical practice. Fam Cancer 18(1): 127-135, 2019. PMID: 29846880. DOI: $10.1007 / \mathrm{s} 10689-018-0089-\mathrm{Z}$

88 Heintz AP, Odicino F, Maisonneuve P, Beller U, Benedet JL, Creasman WT, Ngan HY, Sideri M and Pecorelli S: Carcinoma of the ovary. J Epidemiol Biostat 6(1): 107-38, 2001. PMID: 11385772 .

89 Chalstrey LJ and Benjamin B: High incidence of breast cancer in thyroid cancer patients. Br J Cancer 20(4): 670-675, 1966. PMID: 5964601. DOI: 10.1038/bjc.1966.78

90 Kim C, Bi X, Pan D, Chen Y, Carling T, Ma S, Udelsman R and Zhang Y: The risk of second cancers after diagnosis of primary thyroid cancer is elevated in thyroid microcarcinomas. Thyroid 23(5): 575-582, 2013. PMID: 23237308. DOI: 10.1089/thy.2011. 0406

91 Orosco RK, Hussain T, Brumund KT, Oh DK, Chang DC and Bouvet M: Analysis of age and disease status as predictors of thyroid cancer-specific mortality using the Surveillance, Epidemiology, and End Results database. Thyroid 25(1): 125132, 2015. PMID: 25369076. DOI: 10.1089/thy.2014.0116

92 Guigon CJ, Kim DW, Willingham MC and Cheng SY: Mutation of thyroid hormone receptor- $\beta$ in mice predisposes to the development of mammary tumors. Oncogene 30(30): 33813390, 2011. PMID: 21399657. DOI: 10.1038/onc.2011.50

93 Ni Y, He X, Chen J, Moline J, Mester J, Orloff MS, Ringel MD and Eng C: Germline SDHx variants modify breast and thyroid cancer risks in Cowden and Cowden-like syndrome via FAD/NADdependant destabilization of p53. Hum Mol Genet 21(2): 300-310, 2012. PMID: 21979946. DOI: $10.1093 / \mathrm{hmg} / \mathrm{ddr} 459$
94 Liu L, Shi J, Mao F, Wei J, Fu D and Zhang J: Synchronous primary cancers of the thyroid and breast: A case report and review of the literature department of Thyroid and Breast Surgery, Clinical Medical College of Yangzhou. Oncol Lett 9(1): 351-354, 2015. PMID: 25435991. DOI: 10.3892/ol.2014.2625

95 Yabe N, Murai S, Kunugi C, Nakadai J, Oto I, Yoshikawa T, Kitasato K, Shimizu H, Nakamura A, Masuda A, Miyazaki Y, Ohashi M, Jinno H and Kitagawa Y: Synchronous male bladder cancer and breast cancer - a case report. Gan To Kagaku Ryoho 41(12): 1978-1980, 2014. PMID: 25731395.

96 Parekh JD, Kukrety S, Thandra A and Valenta C: Multiple primary malignant neoplasms in an elderly patient. Cureus 10(3): e2384, 2018. PMID: 29850379. DOI: 10.7759/cureus. 2384

97 Damrauer JS, Hoadley KA, Chism DD, Fan C, Tiganelli CJ, Wobker SE, Yeh JJ, Milowsky MI, Iyer G, Parker JS and Kim WY: Intrinsic subtypes of high-grade bladder cancer reflect the hallmarks of breast cancer biology. Proc Natl Acad Sci USA 111(8): 3110-3115, 2014. PMID: 24520177. DOI: 10.1073/pnas. 1318376111

98 Li FP, Fraumeni JF Jr, Mulvihill JJ, Blattner WA, Dreyfus MG, Tucker MA and Miller RW: A cancer family syndrome in twenty-four families with Li-Fraumeni syndrome. Cancer Res 48: 5358-5362, 1988. PMID: 3409256.

99 Shiseki M, Nishikawa R, Yamamoto H, Ochiai A, Sugimura H, Shitara N, Sameshima Y, Mizoguchi H, Sugimura T and Yokota J: Germ-line p53 mutation is uncommon in patients with triple primary cancers. Cancer Lett 73(1): 51-57, 1993. PMID: 8402598.

100 Hollstein M, Sidransky D, Vogelstein B and Harris CC: p53 mutations in human cancers. Science (Washington DC) 25: 4953, 1991. PMID: 1905840

101 Gallagher RP, Bajdik CD, Fincham S, Hill GB, Keefe AR, Coldman A and McLean DI: Chemical exposures, medical history, and risk of squamous and basal cell carcinoma of the skin. Cancer Epidemiol Biomarkers Prev 5(6): 419-424, 1996. PMID: 8781736.

102 de Oliveira ECV, da Motta VRV, Pantoja PC, Ilha CSO, Magalhães RF, Galadari $\mathrm{H}$ and Leonardi GR: Actinic keratosis - review for clinical practice. Int J Dermatol 58(4): 400-407, 2019. PMID: 30070357. DOI: 10.1111/ijd.14147

103 Munker R, Hiller E, Melnyk A and Gutjahr P: Second malignancies: clinical relevance and basic research. Int J Oncol 9: 763-776, 1996. PMID: 21541581.

104 Vogt A, Schmid S, Heinimann K, Frick H, Herrmann C, Cerny $\mathrm{T}$ and Omlin A: Multiple primary tumours: challenges and approaches, a review. ESMO Open 2(2): e000172, 2017. PMID: 28761745. DOI: 10.1136/esmoopen-2017-000172

105 Pasta V, Mitri F, Amabile MI and Picardi N: Inflammatory breast cancer. Ann Ital Chir 77: 275-279, 2006. PMID: 17137045 